\title{
In Vitro Antimicrobial Activity of Crude Extracts from Vetiveria nigritana (benth.) Stapf, Mitragyna inermis (Willd.) Kuntze, Kalanchoe crenata (andr.) Haw. against Methicillin-resistant Staphylococcus aureus
}

\author{
Roukiatou Traoré ${ }^{1}$, Cheikna Zongo ${ }^{1 *}$, Arouna Ouedraogo ${ }^{1}$, \\ Emmanuel Sampo ${ }^{2}$, Mahamadi Sore ${ }^{3}$, Boubacar Yaro ${ }^{4}$, Yves Traoré ${ }^{1}$ \\ and Aly Savadogo ${ }^{1}$ \\ ${ }^{1}$ Laboratoire de Biochimie et Immunologie Appliquées (LABIA), Université Joseph \\ KI-ZERBO, Burkina Faso \\ ${ }^{2}$ Centre Médical Protestant Schiphra, Burkina Faso \\ ${ }^{3}$ Centre National de Recherche et de Formation sur le Paludisme (CNRFP), Burkina Faso \\ ${ }^{4}$ Institut de Recherche en Sciences de la Santé (IRSS), Burkina Faso \\ *Corresponding Author: Cheikna Zongo, Laboratoire de Biochimie et Immunologie \\ Appliquées (LABIA), Ecole Doctorale Sciences et Technologies, Université Joseph \\ KI-ZERBO, Burkina Faso.
}

Received: March 12, 2021

Published: April 27, 2021

(C) All rights are reserved by Roukiatou

Traore., et al.

\begin{abstract}
The evolution of increasingly antimicrobial-resistant bacterial species in general and particularly. The emergence of strains of methicillin-resistant Staphylococcus aureus (MRSA) are currently a real threat to humanity. There is an urgent need for new efficient antibiotics. Medicinal plants can be the sources of effective new therapeutic agents. This study as performed to study the antimicrobial activity of three medicinal plants (Mitragyna inermis, Vetiveria nigritana, Kalanchoe crenata) from Burkina Faso against Staphylococcus aureus strains isolate from patients. The antibiotics susceptibility of Staphylococcus aureus strains and the antimicrobial activities of the plant extracts were evaluated using standard agar disc diffusion method. Determination of minimum inhibitory concentrations (MIC) and minimum Bactericidal Concentration (MBC) of the active extracts was done using the agar microdilution method. The highest antimicrobial activity was recorded with ethanol extracts of plants against MRSA. No antimicrobial activity was detected with decoction extracts. The MIC and MBC of the different extracts ranged from 0.625 to $10 \mathrm{mg} / \mathrm{ml}$ for Mitragyna inermis and Vetiveria nigritana extracts and from 0.625 to $5 \mathrm{mg} / \mathrm{ml}$ for Kalanchoe crenata extracts.
\end{abstract}

Mitragyna inermis, Vetiveria nigritana, Kalanchoe crenata are some therapeutically potential plants to combat microbial infections due to MRSA.

Keywords: Staphylococcus aureus; Antimicrobial Resistance; MRSA; Mitragyna inermis; Vetiveria nigritana; Kalanchoe crenata; Burkina Faso

\section{Abbreviations}

MRSA: Methicillin-Resistant Staphylococcus aureus; MBC: Minimum Bactericidal Concentration; MIC: Minimum Inhibitory Concentrations; SAB: Staphylococcus aureus Bacteremia; AIDS: Acquired Immunodeficiency Syndrome; CLSI: Clinical Laboratory Standards Institute; DMSO: Dimethyl Sulfoxide; SPSS: Statistical Package for Social Services; DDM: Decoction Extract Dried of $M$. inermis; DDK: Decoction Extract Dried of K. crenata; DDV: Decoction Extract Dried of V. nigritana; EDV: Ethanol Extracts Dried of $V$. nigritana; EDM: Ethanol Extracts Dried of M. inermis; EDK: Ethanol Extracts Dried of K. crenata; ELK: Ethanol Extracts Lyophilized of $K$. crenata; ELV: Ethanol Extracts Lyophilized of V. nigritana; ELM: Ethanol Extracts Lyophilized of M. inermis; DLM: Decoction Extract Lyophilised of M. inermis; DLV: Decoction Extract Lyophilized of $V$. 
nigritana; DLK: Decoction Extract Lyophilised of K. crenata; MRSA/ MDR: Methicillin Resistant Staphylococcus aureus/Multidrug Resistant; DIZ: Diameter of Inhibition Zone; Amox: Amoxicillin; Com Community-acquired S. aureus; Hosp: Hospital-acquired S. aureus

\section{Introduction}

Nowadays, Staphylococcus aureus has been identified as a dangerous and difficult-to-tackle pathogen. Indeed, Staphylococcus aureus is one of the pathogen bacteria with a great capacity to adapt to different environmental conditions and ability to cause a diverse array of life-threatening infections because of its important intrinsic virulence. Besides, it is now, with Clostridium difficile, considered the leading overall cause of hospital acquired infections and is an increasing concern in the community $[1,2]$.

Staphylococcus aureus bacteremia (SAB) is one of the most the important infections due to this bacteria. The incidence of SAB is estimated from 20 to 50 cases/100,000 population per year [3]. Comparatively, the number of deaths due to $\mathrm{SAB}$ is greater than the number due to AIDS, tuberculosis, and viral hepatitis combined [3]. Rates of SAB depend from regions, development status or specific groups of population [4]. In the United States, SAB rate as estimated between 38.2 to 45.7 per 100,000 person-years $[5,6]$. In the industrialized world, the incidence is approximately 10 to 30 per 100,000 person-years [5]. Despite the lack of data, the incidence $\mathrm{SAB}$ can be supposed to be much higher in developing countries and in the poorest regions of the world. This probably could get worse with the rapid expansions of MRSA if nothing is done. The mortality of patients with SAB in the pre-antibiotic era exceeded $80 \%$ and over $70 \%$ developed metastatic infections $[7,8]$. To no longer return to this situation, there is an urgent need to find new antimicrobial agents to deal with antimicrobial resistance and particularly MRSA.

As there is an imperative need to develop new antimicrobial drugs for the treatment of infectious diseases, one approach is to screen local medicinal plants for possible antimicrobial properties [9]. For millennia, medicinal plants have been a valuable source of therapeutic agents [10-12]. Plant secondary metabolites have already demonstrated their potential as antibacterial when used alone and as synergists or potentiators of other antibacterial agents $[13,14]$. Plant extracts or compounds often demonstrate high-level activity against pathogens, and they rarely have severe side effects [15]. Screening local medicinal plants can lead to new efficient antibiotics or complementary and alternative medicine therapies which have been gaining popularity throughout the world $[16,17]$.

The flora of Burkina Faso abounds in a large number of plants used in traditional medicine for the treatment of bacterial infections. Regarding to the traditional use of these plants, some of them could have important therapeutic properties to fight against the multidrug-resistant bacteria like MRSA.

\section{Aim of the Study}

Therefore, the aim of this study was to screen the antibacterial properties of different extracts from three medicinal plants (Mitragyna inermis, Vetiveria nigritana, Kalanchoe crenata) of Burkina Faso against Staphylococcus aureus isolate including MRSA.

\section{Materials and Methods}

Microbial samples and their susceptibility to antimicrobial

The bacteria (40 strains of Staphylococcus aureus) were isolated from patient specimens as described previously [18]. Kirby-Bauer method was performed for antibiotic susceptibility testing according to the guidelines of the Clinical Laboratory Standards Institute [19]. Briefly, fresh bacterial strain with 0.5 McFarland turbidity was swabbed onto the Mueller-Hinton agar (Oxoid, UK) surface using sterile swab sticks. Antimicrobial discs (HIMEDIA, India,) were evenly embedded onto the inoculated agar incubated at $37^{\circ} \mathrm{C}$ overnight. The antibiotics discs used for identification of antibiotic sensitivity pattern of MRSA isolates were: erythromycin $(15 \mu \mathrm{g})$, tetracycline (30 g), gentamicin $(10 \mu \mathrm{g})$, clindamycin $(2 \mu \mathrm{g})$, ciprofloxacin (5 $\mu \mathrm{g})$, levofloxacin (30 $\mu \mathrm{g})$, tobramycin (30 $\mu \mathrm{g})$, kanamycin $(30 \mu \mathrm{g})$, cotrimoxazole $(1.25+23.75 \mu \mathrm{g})$, fusidic acid $(10 \mu \mathrm{g})$, and chloramphenicol $(30 \mu \mathrm{g})$.

\section{Collection and identification of plants}

Plants samples were collected from Loumbila, a village located at the north of Ouagadougou in March 2020. These samples were identified at the Biodiversity Information Centre of University Joseph KI-ZERBO. These plant materials were collected on the basis of their traditional uses by the population as medicines in Burkina Faso. Table 1 show common names and general uses of the three plant species used in this study. 


\begin{tabular}{|c|c|c|c|c|}
\hline Plants names & Common name & Family & Part tested & Traditional uses \\
\hline $\begin{array}{c}\text { Vetiveria nigritana } \\
\text { (Benth.) Stapf }\end{array}$ & Vetiver & Gramineae & Roots & $\begin{array}{c}\text { Boils, epilepsy, burns, snakebites, scorpion stings, fever, } \\
\text { headache, as a tonic for weakness, rheumatism. }\end{array}$ \\
\hline $\begin{array}{c}\text { Mitragyna inermis } \\
\text { (Willd.) Kuntze }\end{array}$ & False abura & Rubiaceae & Leaves & $\begin{array}{c}\text { Malaria, syphilis, bilharzia, gonorrhea, parasitosis, } \\
\text { dysentery and cholera }\end{array}$ \\
\hline $\begin{array}{c}\text { Kalanchoe crenata } \\
\text { (Andr) haw }\end{array}$ & Neverdie & Crassulaceae & Leaves & $\begin{array}{c}\text { Epilepsy, wounds, ear infections, odontalgia, burns, } \\
\text { ulcers }\end{array}$ \\
\hline
\end{tabular}

Table 1: Selected medicinal plants the parts used and their traditional uses.

After harvesting, the samples were washed under running water to remove dust. Subsequently, they were dried in the shade, and afterwards the dried plant materials were finely grounded by mechanical grinders. The powder was stored in tightly closed glass containers in the dark at room temperature.

\section{Preparation of plant extracts}

The ethanol extracts were prepared by soaking $25 \mathrm{~g}$ of the fine powder of each plant part was in $250 \mathrm{ml}$ of ethanol (70\%) with stirring for $48 \mathrm{~h}$. Then, the extracts were firstly filtered through double layers of compress and finally filtered again through Whatman no. 1 filter paper. The ethanol was evaporated using rotatory vacuum evaporator and each filtrate was divided into 2 parts. A part was concentrated then dried in an oven at $40^{\circ} \mathrm{C}$ under ventilation. The other part was freezed and lyophilized. For each plant, a dried extract and a lyophilized extract were obtained.

The water extracts were obtained by boiling $25 \mathrm{~g}$ of the powder of each plant part in distilled water for 30 minutes. This process follows approximately that of traditional healer's method. Each decoction is then cooled, filtered and concentrated with a rotary evaporator under vacuum. Each filtrate was treated as described above to obtain dried decocted extract and lyophilized decocted extract.

Crude extracts were weighted and stored in small bottles in fridge at $5^{\circ} \mathrm{C}$ and their yield calculated using the following equation:

$$
\text { Yield }(\mathrm{g} / 100 \mathrm{~g})=\frac{\mathrm{w}_{1} \times 100}{\mathrm{w}_{2}}
$$

Where W1 is the weight of the extract residue obtained after drying of lyophilisation and $\mathrm{W} 2$ is the weight of the plant part powder used for the extraction.

\section{Plants antibacterial activity evaluation}

In the antibacterial tests, Kirby-Bauer method was performed according to the Clinical Laboratory Standards Institute guidelines
[19]. Extracts were dissolved in dimethyl sulfoxide (DMSO) to obtain final concentration of $100 \mathrm{mg} / \mathrm{ml}$ and sterilized through Millipore filter $(0.22 \mathrm{~mm})$. Then $10 \mu 1$ were loaded over sterile filter paper discs (6 $\mathrm{mm}$ in diameter) Disks are left under the hood for a few minutes at room temperature.

An overnight culture of each strain with $0.5 \mathrm{McFarland}$ turbidity was swabbed onto the Mueller-Hinton agar (Oxoid, UK) surface using sterile swab sticks. The paper discs prepared with the extracts were then placed onto the inoculated agar incubated at $37^{\circ} \mathrm{C}$ for 24h. Antibiotic discs (Amoxicillin) was used as positive control and paper discs soaked in DMSO without extract were used as negative control (DMSO didn't show inhibition effects to the growth of bacterial strains used).

Antibacterial activity is recorded when an inhibition zone diameter more than $9 \mathrm{~mm}$ is observed around the paper disk [20]. The inhibition zone diameters were measured with vernier calliper.

Determination of minimum inhibitory concentration (MIC) and minimum bactericidal concentration (MBC) of active extracts

Determination of minimum inhibitory concentrations (MIC) and minimum Bactericidal Concentration (MBC) of the extracts was done using the agar microdilution method [21]. The extracts that showed antimicrobial activity were selected to determine the MIC and the MBC for Staphylococcus aureus strains. Seven strains (1 SASM, 2 SARM, 3 SARM/MDR, and 1 SR) were selected and grown in nutrient broth for theses assays.

Each previously prepared and sterilized plant extract which showed antibacterial activity in the previous test was transferred in sterile 96 wells-plates (Nunc) previously filed with sterile nutrient broth to obtain a serial dilutions ranging from 20 to 0.0975 $\mathrm{mg} / \mathrm{ml}$. Then plates were inoculated with microbial suspensions diluted from the same 0.5 MacFarland standards to have 1 to $2 \mathrm{x}$ $10^{6} \mathrm{CFU} /$ in each well. Some wells were reserved in each 96 wells plate for sterility control (no microorganism added), inoculums vi- 
ability (no extract added) and the DMSO inhibitory effect. The final volumes in wells were $200 \mu \mathrm{L}$. Afterwards $24 \mathrm{~h}$ at $37^{\circ} \mathrm{C}$, the $\mathrm{MIC}$ of each sample was appreciated visually by appreciating the growth of the microorganisms in each well (presence or absence of turbidity and/or a pellet in the well) with comparison to sterile and noninoculated nutrient broth. The MIC is the lowest concentration of antimicrobial agent that completely inhibits growth of the studied microorganism. The experiment was replicated 3 times.

To determine the $\mathrm{MBC}$, the microbial suspension was taken from the wells of concentration greater than or equal to MIC and inoculated on the Mueller-Hinton agar and then incubated for 24 hours at $37^{\circ} \mathrm{C}$. The lowest extracts concentration at which no growth was observed on the agar after $24 \mathrm{~h}$ of incubation is considered as MBC. The MBC was defined as the lowest extract concentration at which $99.9 \%$ of the bacteria have been killed. The experiment was replicated 3 times. The ratio MBC/ MIC was used to determine the intrinsic activity (bactericidal or bacteriostatic) of plant extracts considering that:

- When MBC/MIC $=1$ or $1<\mathrm{MBC} / \mathrm{MIC} \leq 4$, it shows absolute bactericidal activity.

- When $8<\mathrm{MBC} / \mathrm{MIC}<16$, it shows bacteriostatic activity.

Data analysis

The data were described as mean standard deviation (S.D.) using SPSS for Windows version 20. (Statistical Package for Social Services, Chicago, IL, USA).

\section{Results}

\section{Extraction yields}

The yields of the studied extracts are summarised in table 2 . There was no significant difference between yields of aqueous extracts and ethanol extracts. Yields varied with plant used. The extracts yielded from 0.25 to $2.72 \%$. The highest yield of plant extract was obtained from Kalanchoe crenata residue dried (2.72\%) followed by Mitragyna inermis (2.63\%) while Vetiveria nigritana give the lowest extract yield respectively (Table 2).

Susceptibility to antibiotics of tested Staphylococcus aureus strains

Out of the 40 S. aureus strains selected and tested, 9 (22.5\%) were MRSA and 20 (50\%) were Staphylococcus aureus MethicillinResistant-Multidrug resistant (MRSA/MDR) (Table 3). Multidrug resistance was defined as a resistance to at least one agent in three or more antimicrobial. It can be noticed the high level of MRSA/ MDR among tested $S$. aureus strains.

\begin{tabular}{|c|c|}
\hline Plant extracts & Yield(\%) \\
\hline DDM & 1.70 \\
\hline DDK & 2.72 \\
\hline DDV & 0.27 \\
\hline EDV & 0.72 \\
\hline EDM & 2.63 \\
\hline EDK & 2.17 \\
\hline ELK & 1.18 \\
\hline ELV & 0.87 \\
\hline ELM & 1.92 \\
\hline DLM & 1.54 \\
\hline DLV & 0.85 \\
\hline DLK & 2.59 \\
\hline
\end{tabular}

Table 2: Plant extracts yield percentage.

DDM: Decoction Extract Dried of M. inermis; DDK: decoction extract dried of $K$. crenata; DDV: Decoction Extract Dried of V. nigritana; EDV: Ethanol Extracts Dried of V. nigritana; EDM: Ethanol Extracts Dried of M. inermis; EDK: Ethanol Extracts Dried of K. crenata; ELK: Ethanol Extracts Lyophilized of $K$. crenata; ELV: Ethanol Extracts Lyophilized of V. nigritana. ELM: Ethanol Extracts Lyophilized of $M$. inermis; DLM: Decoction Extract Lyophilised of M. inermis; DLV: Decoction Extract Lyophilized of V. nigritana; DLK: Decoction Extract Lyophilised of $K$. crenata.

\begin{tabular}{|c|c|}
\hline Antibiotic sensitivity & Number of strain (N (\%)) \\
\hline MSSA & $11(27.5)$ \\
\hline MRSA & $9(22.5)$ \\
\hline MRSA/MDR & $20(50)$ \\
\hline
\end{tabular}

Table 3: Antibiotic sensitivity pattern of Staphylococcus aureus isolates.

MRSA: Methicillin Resistant Staphylococcus aureus; MRSA/ MDR: Methicillin Resistant Staphylococcus aureus/Multidrug Resistant.

Antibacterial activity of plant extracts

The results of our investigations to evaluate the antibacterial activity of the 3 medicinal plants against Staphylococcus aureus strains showed variable antimicrobial activity. Al the 3 plants exhibited inhibitory effect against studied microorganisms but this effect depend on the type of extract.

It can be seen that ethanol extract of Mitragyna inermis gave larger diameter of inhibition zone (DIZ) than aqueous extract (Table 4). Decocted and oven-dried extract did not show antibacterial 
In Vitro Antimicrobial Activity of Crude Extracts from Vetiveria nigritana (benth.) Stapf, Mitragyna inermis (Willd.) Kuntze, Kalanchoe crenata (andr.) Haw. against Methicillin-resistant Staphylococcus aureus

\begin{tabular}{|c|c|c|c|c|c|c|c|}
\hline \multirow{2}{*}{ Strains code } & \multirow{2}{*}{ Origin } & \multirow{2}{*}{ Strain type } & \multicolumn{2}{|c|}{ Decoction extracts } & \multicolumn{2}{|c|}{ Ethanol extracts } & \multirow{2}{*}{ Amox } \\
\hline & & & Oven-dried & lyophilised & Oven-dried & lyophilised & \\
\hline SA1 & com & MRSA/MDR & $6 \pm 0.00$ & $7 \pm 1.44$ & $9.5 \pm 0.72$ & $11 \pm 0.72$ & $14 \pm 1.44$ \\
\hline SA2 & hosp & MRSA/MDR & $6 \pm 0.00$ & $7 \pm 1.44$ & $10 \pm 0.00$ & $11.5 \pm 0.72$ & $9 \pm 1.44$ \\
\hline SA3 & hosp & MRSA/MDR & $6 \pm 0.00$ & $8 \pm 0.00$ & $11 \pm 0.00$ & $12 \pm 0.00$ & $11.5 \pm 0.72$ \\
\hline SA4 & com & MRSA/MDR & $6 \pm 0.00$ & $9 \pm 0.00$ & $10.5 \pm 0.72$ & $12 \pm 0.00$ & $8.5 \pm 0.72$ \\
\hline SA5 & hosp & MRSA/MDR & $6 \pm 0.00$ & $9 \pm 0.00$ & $10 \pm 0.00$ & $12 \pm 0.00$ & $14.5 \pm 0.72$ \\
\hline SA6 & hop & MRSA/MDR & $6 \pm 0.00$ & $9 \pm 0.00$ & $11 \pm 0.00$ & $12 \pm 0.00$ & $10.5 \pm 0.72$ \\
\hline SA7 & com & MRSA/MDR & $6 \pm 0.00$ & $6 \pm 0.00$ & $10.5 \pm 0.72$ & $12.5 \pm 0.72$ & $14.5 \pm 0.72$ \\
\hline SA8 & com & MRSA/MDR & $6 \pm 0.00$ & $6 \pm 0.00$ & $10 \pm 0.00$ & $10.5 \pm 0.72$ & $10 \pm 0.00$ \\
\hline SA9 & com & MRSA/MDR & $6 \pm 0.00$ & $7 \pm 0.00$ & $10 \pm 0.00$ & $12 \pm 0.72$ & $9.5 \pm 0.72$ \\
\hline SA10 & com & MRSA/MDR & $6 \pm 0.00$ & $9 \pm 0.00$ & $11 \pm 0.00$ & $12.5 \pm 0.72$ & $8 \pm 0.00$ \\
\hline SA11 & com & MRSA/MDR & $6 \pm 0.00$ & $8.5 \pm 0.72$ & $10.5 \pm 0.72$ & $11 \pm 0.00$ & $15 \pm 1.4$ \\
\hline SA12 & hosp & MRSA/MDR & $6 \pm 0.00$ & $6 \pm 0.00$ & $10 \pm 0.00$ & $11.5 \pm 0.72$ & $13 \pm 1.4$ \\
\hline SA13 & com & MRSA/MDR & $6 \pm 0.00$ & $9 \pm 0.00$ & $11 \pm 0.00$ & $11.5 \pm 0.72$ & $14.5 \pm 0.72$ \\
\hline SA14 & com & MSSA & $6 \pm 0.00$ & $6 \pm 0.00$ & $10.5 \pm 0.72$ & $12 \pm 0.00$ & $6 \pm 0.00$ \\
\hline SA15 & com & MSSA & $6 \pm 0.00$ & $9 \pm 0.00$ & $11 \pm 0.00$ & $11.5 \pm 0.72$ & $10.5 \pm 0.72$ \\
\hline SA16 & com & MRSA/MDR & $6 \pm 0.00$ & $8.5 \pm 0.72$ & $10 \pm 0.00$ & $12.5 \pm 0.72$ & $14 \pm 1.44$ \\
\hline SA17 & com & MRSA/MDR & $6 \pm 0.00$ & $6 \pm 0.00$ & $10 \pm 0.00$ & $11.5 \pm 0.72$ & $12 \pm 0.00$ \\
\hline SA18 & com & MSSA & $6 \pm 0.00$ & $9.5 \pm 0.72$ & $10 \pm 0.00$ & $11.5 \pm 0.72$ & $13.5 \pm 0.72$ \\
\hline SA19 & hosp & MRSA & $6 \pm 0.00$ & $6 \pm 0.00$ & $10 \pm 0.00$ & $12.5 \pm 0.72$ & $14.5 \pm 0.72$ \\
\hline SA20 & hosp & MSSA & $6 \pm 0.00$ & $6.5 \pm 0.72$ & $11.5 \pm 0.72$ & $11 \pm 0.00$ & $14 \pm 0.00$ \\
\hline SA21 & com & MSSA & $6 \pm 0.00$ & $9.5 \pm 0.72$ & $11.5 \pm 0.72$ & $12.5 \pm 0.72$ & $10.5 \pm 0.72$ \\
\hline SA22 & hosp & MRSA/MDR & $6 \pm 0.00$ & $6.5 \pm 0.72$ & $10.5 \pm 0.72$ & $12.5 \pm 0.72$ & $23.5 \pm 0.72$ \\
\hline SA23 & hosp & MRSA/MDR & $6 \pm 0.00$ & $6.5 \pm 0.72$ & $9 \pm 1.44$ & $11.5 \pm 0.72$ & $14.5 \pm 0.72$ \\
\hline SA24 & com & MRSA & $6 \pm 0.00$ & $9.5 \pm 0.72$ & $10.5 \pm 0.72$ & $11.5 \pm 0.72$ & $30 \pm 0.00$ \\
\hline SA25 & com & MRSA & $6 \pm 0.00$ & $8 \pm 0.00$ & $10 \pm 0.00$ & $12.5 \pm 0.72$ & $12 \pm 0.00$ \\
\hline SA26 & hosp & MSSA & $6 \pm 0.00$ & $9.5 \pm 0.72$ & $11.5 \pm 0.00$ & $11.5 \pm 0.72$ & $12.5 \pm 0.72$ \\
\hline SA27 & hosp & MSSA & $6 \pm 0.00$ & $7 \pm 0.00$ & $9 \pm 0.00$ & $12 \pm 0.00$ & $32 \pm 1.44$ \\
\hline SA28 & com & MRSA & $6 \pm 0.00$ & $8 \pm 0.00$ & $11 \pm 1.44$ & $11.5 \pm 0.72$ & $13 \pm 1.44$ \\
\hline SZ29 & com & MRSA & $6 \pm 0.00$ & $9 \pm 1.44$ & $11.5 \pm 0.72$ & $12 \pm 0.00$ & $145 \pm 0.72$ \\
\hline SA30 & com & MRSA & $6 \pm 0.00$ & $7 \pm 0.00$ & $12 \pm 0.00$ & $11.5 \pm 0.72$ & $11 \pm 1.44$ \\
\hline SA31 & hosp & MSSA & $6 \pm 0.00$ & $6 \pm 0.00$ & $10.5 \pm 0.72$ & $11.5 \pm 0.72$ & $11 \pm 1.44$ \\
\hline SA32 & hosp & MRSA & $6 \pm 0.00$ & $7 \pm 0.00$ & $9 \pm 0.00$ & $10.5 \pm 0.72$ & $6 \pm 0.00$ \\
\hline SA33 & hosp & MRSA & $6 \pm 0.00$ & $6 \pm 0.00$ & $10 \pm 0.00$ & $10.5 \pm 0.72$ & $15.5 \pm 0.72$ \\
\hline SA34 & hosp & MRSA & $6 \pm 0.00$ & $6 \pm 0.00$ & $10.5 \pm 0.72$ & $10.5 \pm 0.72$ & $13.5 \pm 0.72$ \\
\hline SA35 & hosp & MRSA/MDR & $6 \pm 0.00$ & $7 \pm 0.00$ & $9.5 \pm 0.72$ & $11.5 \pm 0.72$ & $14.5 \pm 0.72$ \\
\hline SA36 & hosp & MSSA & $6 \pm 0.00$ & $6 \pm 0.00$ & $10 \pm 0.00$ & $12.5 \pm 0.72$ & $15.5 \pm 0.72$ \\
\hline SA37 & com & MSSA & $6 \pm 0.00$ & $7 \pm 0.00$ & $8.5 \pm 0.72$ & $11.5 \pm 0.72$ & $29.5 \pm 0.72$ \\
\hline SA38 & com & MSSA & $6 \pm 0.00$ & $7 \pm 0.00$ & $10 \pm 1.44$ & $12.5 \pm 0.72$ & $13 \pm 1.44$ \\
\hline SA39 & hosp & MRSA/MDR & $6 \pm 0.00$ & $6.5 \pm 0.72$ & $11.5 \pm 0.72$ & $12 \pm 0.00$ & $14.5 \pm 0.72$ \\
\hline SA40 & hosp & MRSA/MDR & $6 \pm 0.00$ & $6 \pm 0.00$ & $9 \pm 0.00$ & $10 \pm 0.00$ & $11 \pm 1.44$ \\
\hline S. aureus ATCC 25923 & - & MSSA & $6 \pm 0.00$ & $6.5 \pm 0.72$ & $10.5 \pm 0.72$ & $12 \pm 1.44$ & $31 \pm 1.44$ \\
\hline
\end{tabular}

Table 4: Diameters of inhibition zone (mm) of Mitragyna inermis extracts.

Amox: Amoxicillin; com: Community-acquired S. aureus; hosp: Hospital-acquired S. aureus. 
In Vitro Antimicrobial Activity of Crude Extracts from Vetiveria nigritana (benth.) Stapf, Mitragyna inermis (Willd.) Kuntze, Kalanchoe crenata (andr.) Haw. against Methicillin-resistant Staphylococcus aureus

activity in this study. Lyophilized extracts (ethanol and aqueous) showed better activity against MRSA/MDR and MRSA. The higher DIZ obtained with $M$. inermis ethanol extract on either MRSA/MDA and MSSA was $12 \mathrm{~mm}$.
As observed with the extracts of Mitragyna inermis, the aqueous extracts of Vetiveria nigritana (Table 5) and those of Kalanchoe crenata (Table 6) did not show antibacterial activity against $S$. aureus strains in this study. On the other hand, their ethanol extracts

\begin{tabular}{|c|c|c|c|c|c|c|c|}
\hline \multirow{2}{*}{ Strains } & \multirow{2}{*}{ Origin } & \multirow{2}{*}{ Strain type } & \multicolumn{2}{|c|}{ Decoction extracts } & \multicolumn{2}{|c|}{ Ethanol extracts } & \multirow{2}{*}{ Amox } \\
\hline & & & Oven-dried & Lyophilised & Oven-dried & Lyophilised & \\
\hline SA1 & com & MRSA/MDR & $6 \pm 0.00$ & $6 \pm 0.00$ & $12.5 \pm 0.72$ & $9 \pm 0.00$ & $14 \pm 1.44$ \\
\hline SA2 & hosp & MRSA/MDR & $6 \pm 0.00$ & $6 \pm 0.00$ & $10 \pm 1.44$ & $8 \pm 1.44$ & $9 \pm 1.44$ \\
\hline SA3 & hosp & MRSA/MDR & $6 \pm 0.00$ & $6 \pm 0.00$ & $10 \pm 0.00$ & $7 \pm 0.00$ & $11.5 \pm 0.72$ \\
\hline SA4 & com & MRSA/MDR & $6 \pm 0.00$ & $6 \pm 0.00$ & $10.5 \pm 0.72$ & $7 \pm 0.00$ & $8.5 \pm 0.72$ \\
\hline SA5 & hosp & MRSA/MDR & $6 \pm 0.00$ & $6 \pm 0.00$ & $9.5 \pm 0.72$ & $6.5 \pm 0.72$ & $14.5 \pm 0.72$ \\
\hline SA6 & hop & MRSA/MDR & $6 \pm 0.00$ & $6 \pm 0.00$ & $9.5 \pm 0.72$ & $9 \pm 0.00$ & $10.5 \pm 0.72$ \\
\hline SA7 & com & MRSA/MDR & $6 \pm 0.00$ & $6 \pm 0.00$ & $10 \pm 0.00$ & $7 \pm 0.00$ & $14.5 \pm 0.72$ \\
\hline SA8 & com & MRSA/MDR & $6 \pm 0.00$ & $6 \pm 0.00$ & $10 \pm 0.00$ & $7 \pm 0.00$ & $10 \pm 0.00$ \\
\hline SA9 & com & MRSA/MDR & $6 \pm 0.00$ & $6 \pm 0.00$ & $10 \pm 0.00$ & $9.5 \pm 0.72$ & $9.5 \pm 0.72$ \\
\hline SA10 & com & MRSA/MDR & $6 \pm 0.00$ & $6 \pm 0.00$ & $10 \pm 0.00$ & $9 \pm 0.00$ & $8 \pm 0.00$ \\
\hline SA11 & com & MRSA/MDR & $6 \pm 0.00$ & $6 \pm 0.00$ & $9.5 \pm 0.72$ & $9 \pm 0.00$ & $15 \pm 1.4$ \\
\hline SA12 & hosp & MRSA/MDR & $6 \pm 0.00$ & $6 \pm 0.00$ & $11 \pm 0.00$ & $9.5 \pm 1.72$ & $13 \pm 1.4$ \\
\hline SA13 & com & MRSA/MDR & $6 \pm 0.00$ & $6 \pm 0.00$ & $10 \pm 0.00$ & $9 \pm 0.00$ & $14.5 \pm 0.72$ \\
\hline SA14 & com & MSSA & $6 \pm 0.00$ & $6 \pm 0.00$ & $12.5 \pm 0.72$ & $11.5 \pm 0.72$ & $6 \pm 0.00$ \\
\hline SA15 & com & MSSA & $6 \pm 0.00$ & $6 \pm 0.00$ & $11.5 \pm 0.72$ & $10 \pm 0.00$ & $10.5 \pm 0.72$ \\
\hline SA16 & com & MRSA/MDR & $6 \pm 0.00$ & $6 \pm 0.00$ & $10.5 \pm 0.72$ & $9.5 \pm 0.72$ & $14 \pm 1.44$ \\
\hline SA17 & com & MRSA/MDR & $6 \pm 0.00$ & $6 \pm 0.00$ & $10.5 \pm 0.72$ & $7.5 \pm 2.1$ & $12 \pm 0.00$ \\
\hline SA18 & com & MSSA & $6 \pm 0.00$ & $6 \pm 0.00$ & $10.5 \pm 0.72$ & $6.5 \pm 0.72$ & $13.5 \pm 0.72$ \\
\hline SA19 & hosp & MRSA & $6 \pm 0.00$ & $6 \pm 0.00$ & $8 \pm 0.00$ & $8.5 \pm 0.72$ & $14.5 \pm 0.72$ \\
\hline SA20 & hosp & MSSA & $6 \pm 0.00$ & $6 \pm 0.00$ & $9.5 \pm 0.72$ & $11.5 \pm 0.72$ & $14 \pm 0.00$ \\
\hline SA21 & com & MSSA & $6 \pm 0.00$ & $6 \pm 0.00$ & $10 \pm 0.00$ & $10 \pm 0.00$ & $10.5 \pm 0.72$ \\
\hline SA22 & hosp & MRSA/MDR & $6 \pm 0.00$ & $6 \pm 0.00$ & $8.5 \pm 0.72$ & $10.5 \pm 0.72$ & $23.5 \pm 0.72$ \\
\hline SA23 & hosp & MRSA/MDR & $6 \pm 0.00$ & $6 \pm 0.00$ & $9 \pm 0.00$ & $9.8 \pm 0.72$ & $14.5 \pm 0.72$ \\
\hline SA24 & com & MRSA & $6 \pm 0.00$ & $6 \pm 0.00$ & $11 \pm 0.00$ & $10 \pm 2.88$ & $30 \pm 0.00$ \\
\hline SA25 & com & MRSA & $6 \pm 0.00$ & $6 \pm 0.00$ & $8 \pm 0.00$ & $11 \pm 0.00$ & $12 \pm 0.00$ \\
\hline SA26 & hosp & MSSA & $6 \pm 0.00$ & $6 \pm 0.00$ & $13.5 \pm 0.72$ & $10 \pm 1.44$ & $12.5 \pm 0.72$ \\
\hline SA27 & hosp & MSSA & $6 \pm 0.00$ & $6 \pm 0.00$ & $11 \pm 2.88$ & $12 \pm 1.44$ & $32 \pm 1.44$ \\
\hline SA28 & com & MRSA & $6 \pm 0.00$ & $6 \pm 0.00$ & $6.5 \pm 0.72$ & $7 \pm 1.44$ & $13 \pm 1.44$ \\
\hline SZ29 & com & MRSA & $6 \pm 0.00$ & $6 \pm 0.00$ & $10 \pm 0.00$ & $11.5 \pm 0.72$ & $145 \pm 0.72$ \\
\hline SA30 & com & MRSA & $6 \pm 0.00$ & $6 \pm 0.00$ & $11 \pm 0.00$ & $9.5 \pm 0.72$ & $11 \pm 1.44$ \\
\hline SA31 & hosp & MSSA & $6 \pm 0.00$ & $6 \pm 0.00$ & $10.5 \pm 0.72$ & $10.5 \pm 0.72$ & $11 \pm 1.44$ \\
\hline SA32 & hosp & MRSA & $6 \pm 0.00$ & $6 \pm 0.00$ & $10.5 \pm 0.72$ & $12.5 \pm 0.72$ & $6 \pm 0.00$ \\
\hline SA33 & hosp & MRSA & $6 \pm 0.00$ & $6 \pm 0.00$ & $7.5 \pm 0.72$ & $12.5 \pm 2.16$ & $15.5 \pm 0.72$ \\
\hline SA34 & hosp & MRSA & $6 \pm 0.00$ & $6 \pm 0.00$ & $8.5 \pm 0.72$ & $10.5 \pm 0.72$ & $13.5 \pm 0.72$ \\
\hline SA35 & hosp & MRSA/MDR & $6 \pm 0.00$ & $6 \pm 0.00$ & $8.5 \pm 0.72$ & $10.5 \pm 0.72$ & $14.5 \pm 0.72$ \\
\hline SA36 & hosp & MSSA & $6 \pm 0.00$ & $6 \pm 0.00$ & $8 \pm 0.00$ & $10.5 \pm 0.72$ & $15.5 \pm 0.72$ \\
\hline
\end{tabular}


In Vitro Antimicrobial Activity of Crude Extracts from Vetiveria nigritana (benth.) Stapf, Mitragyna inermis (Willd.) Kuntze, Kalanchoe crenata (andr.) Haw. against Methicillin-resistant Staphylococcus aureus

\begin{tabular}{|c|c|c|c|c|c|c|c|}
\hline SA37 & com & MSSA & $6 \pm 0.00$ & $6 \pm 0.00$ & $9.5 \pm 2.16$ & $11 \pm 1.44$ & $29.5 \pm 0.72$ \\
\hline SA38 & com & MSSA & $6 \pm 0.00$ & $6 \pm 0.00$ & $8.5 \pm 0.7$ & $11.5 \pm 0.72$ & $13 \pm 1.44$ \\
\hline SA39 & hosp & MRSA/MDR & $6 \pm 0.00$ & $6 \pm 0.00$ & $9 \pm 0.00$ & $12.5 \pm 0.72$ & $14.5 \pm 0.72$ \\
\hline SA40 & hosp & MRSA/MDR & $6 \pm 0.00$ & $6 \pm 0.00$ & $8 \pm 1.44$ & $11.5 \pm 0.72$ & $11 \pm 1.44$ \\
\hline S. aureus ATCC 25923 & - & MSSA & $6 \pm 0.00$ & $6 \pm 0.00$ & $9 \pm 0.00$ & $10 \pm 1.44$ & $31 \pm 1.44$ \\
\hline
\end{tabular}

Table 5: Diameters of inhibition zone ( $\mathrm{mm}$ ) of Vetiveria nigritana extracts.

Amox: Amoxicillin; com: Community-acquired S. aureus; osp: hospital-acquired S. aureus.

showed moderate antibacterial activity. There was no significant difference in the antibacterial activity for MRSA/MDR, MRSA or MSSA. The DIZ obtained with plant extracts were more or less comparable to those obtained with the reference antibiotics used. However, it should be noted that the extract disc loads were of the order of milligrams while those of the antibiotics had loads of the order of micrograms.
Minimum inhibitory concentrations (MIC) and minimum bactericidal concentrations (MBC) of the active plant extracts

The MIC and the MBC of the most effective plant extracts (ethanol extracts) were determined by microdilution method as described above. For Mitragyna inermis ethanol extracts, MIC ranged from 0.625 to $2.5 \mathrm{mg} / \mathrm{ml}$ and MBC from 5 to $10 \mathrm{mg} / \mathrm{ml}$ (Table 7). Similar results were recorded with the ethanol extracts of Vetiveria

\begin{tabular}{|c|c|c|c|c|c|c|c|}
\hline \multirow{2}{*}{ Strains } & \multirow{2}{*}{ Origin } & \multirow{2}{*}{ Strain type } & \multicolumn{2}{|c|}{ Decoction extracts } & \multicolumn{2}{|c|}{ Ethanol extracts } & \multirow{2}{*}{ Amox } \\
\hline & & & Oven-dried & Lyophilised & Oven-dried & Lyophilised & \\
\hline SA1 & com & MRSA/MDR & $6 \pm 0.00$ & $9 \pm 0.00$ & $12 \pm 1.44$ & $10.5 \pm 0.72$ & $14 \pm 1.44$ \\
\hline SA2 & hosp & MRSA/MDR & $6 \pm 0.00$ & $9.5 \pm 0.00$ & $11.5 \pm 0.72$ & $11.5 \pm 0.72$ & $9 \pm 1.44$ \\
\hline SA3 & hosp & MRSA/MDR & $6 \pm 0.00$ & $9.5 \pm 0.72$ & $11 \pm 0.00$ & $12 \pm 0.00$ & $11.5 \pm 0.72$ \\
\hline SA4 & com & MRSA/MDR & $6 \pm 0.00$ & $10.5 \pm 0.72$ & $12 \pm 0.00$ & $12.5 \pm 0.72$ & $8.5 \pm 0.72$ \\
\hline SA5 & hosp & MRSA/MDR & $6 \pm 0.00$ & $7.5 \pm 2.16$ & $12 \pm 0.00$ & $10.5 \pm 0.72$ & $14.5 \pm 0.72$ \\
\hline SA6 & hosp & MRSA/MDR & $6 \pm 0.00$ & $9 \pm 0.00$ & $11.5 \pm 0.72$ & $12 \pm 0.00$ & $10.5 \pm 0.72$ \\
\hline SA7 & com & MRSA/MDR & $6 \pm 0.00$ & $10 \pm 1.44$ & $12 \pm 0.00$ & $11.5 \pm 0.72$ & $14.5 \pm 0.72$ \\
\hline SA8 & com & MRSA/MDR & $6 \pm 0.00$ & $6 \pm 0.00$ & $11.5 \pm 0.72$ & $11 \pm 0.72$ & $10 \pm 0.00$ \\
\hline SA9 & com & MRSA/MDR & $6 \pm 0.00$ & $7 \pm 1.44$ & $11 \pm 0.00$ & $11.5 \pm 0.72$ & $9.5 \pm 0.72$ \\
\hline SA10 & com & MRSA/MDR & $6 \pm 0.00$ & $9.5 \pm 0.72$ & $11 \pm 0.00$ & $12.5 \pm 0.72$ & $8 \pm 0.00$ \\
\hline SA11 & com & MRSA/MDR & $6 \pm 0.00$ & $8.5 \pm 0.72$ & $11.5 \pm 0.72$ & $11 \pm 0.00$ & $15 \pm 1.4$ \\
\hline SA12 & hosp & MRSA/MDR & $6 \pm 0.00$ & $7 \pm 0.00$ & $12 \pm 0.00$ & $11 \pm 0.00$ & $13 \pm 1.4$ \\
\hline SA13 & com & MRSA/MDR & $6 \pm 0.00$ & $9 \pm 0.00$ & $11 \pm 0.00$ & $12 \pm 0.00$ & $14.5 \pm 0.72$ \\
\hline SA14 & com & MSSA & $6 \pm 0.00$ & $8 \pm 0.00$ & $12.5 \pm 0.72$ & $12 \pm 1.4$ & $6 \pm 0.00$ \\
\hline SA15 & com & MSSA & $6 \pm 0.00$ & $7 \pm 0.00$ & $10.5 \pm 0.72$ & $11 \pm 0.00$ & $10.5 \pm 0.72$ \\
\hline SA16 & com & MRSA/MDR & $6 \pm 0.00$ & $8.5 \pm 0.00$ & $11.5 \pm 0.72$ & $11.5 \pm 0.72$ & $14 \pm 1.44$ \\
\hline SA17 & com & MRSA/MDR & $6 \pm 0.00$ & $9 \pm 0.00$ & $11 \pm 0.00$ & $11.5 \pm 0.72$ & $12 \pm 0.00$ \\
\hline SA18 & com & MSSA & $6 \pm 0.00$ & $6.5 \pm 0.72$ & $18 \pm 0.00$ & $11.5 \pm 0.72$ & $13.5 \pm 0.72$ \\
\hline SA19 & hosp & MRSA & $6 \pm 0.00$ & $10 \pm 0.00$ & $13.5 \pm 2.1$ & $12.5 \pm 0.72$ & $14.5 \pm 0.72$ \\
\hline SA20 & hosp & MSSA & $6 \pm 0.00$ & $10 \pm 0.00$ & $11.5 \pm 0.72$ & $11 \pm 1.4$ & $14 \pm 0.00$ \\
\hline SA21 & com & MSSA & $6 \pm 0.00$ & $8.5 \pm 0.72$ & $11.5 \pm 0.72$ & $12.5 \pm 0.72$ & $10.5 \pm 0.72$ \\
\hline SA22 & hosp & MRSA/MDR & $6 \pm 0.00$ & $9 \pm 0.00$ & $11.5 \pm 0.72$ & $11.5 \pm 0.72$ & $23.5 \pm 0.72$ \\
\hline SA23 & hosp & MRSA/MDR & $6 \pm 0.00$ & $6.5 \pm 0.72$ & $9.5 \pm 0.72$ & $11.5 \pm 0.72$ & $14.5 \pm 0.72$ \\
\hline SA24 & com & MRSA & $6 \pm 0.00$ & $10 \pm 0.00$ & $11.5 \pm 0.72$ & $12.5 \pm 0.72$ & $30 \pm 0.00$ \\
\hline SA25 & com & MRSA & $6 \pm 0.00$ & $8.5 \pm 0.72$ & $12.5 \pm 0.72$ & $11.5 \pm 0.72$ & $12 \pm 0.00$ \\
\hline
\end{tabular}


In Vitro Antimicrobial Activity of Crude Extracts from Vetiveria nigritana (benth.) Stapf, Mitragyna inermis (Willd.) Kuntze, Kalanchoe crenata (andr.) Haw. against Methicillin-resistant Staphylococcus aureus

\begin{tabular}{|c|c|c|c|c|c|c|c|}
\hline SA26 & hosp & MSSA & $6 \pm 0.00$ & $9.5 \pm 0.72$ & $10.5 \pm 0.72$ & $11.5 \pm 0.72$ & $12.5 \pm 0.72$ \\
\hline SA27 & hosp & MSSA & $6 \pm 0.00$ & $8 \pm 0.00$ & $12.5 \pm 2.16$ & $11 \pm 0.00$ & $32 \pm 1.44$ \\
\hline SA28 & com & MRSA & $6 \pm 0.00$ & $10 \pm 0.00$ & $11 \pm 0.00$ & $13 \pm 1.4$ & $13 \pm 1.44$ \\
\hline SZ29 & com & MRSA & $6 \pm 0.00$ & $10.5 \pm 0.72$ & $12.5 \pm 0.72$ & $14.5 \pm 0.72$ & $145 \pm 0.72$ \\
\hline SA30 & com & MRSA & $6 \pm 0.00$ & $10 \pm 0.00$ & $11 \pm 0.00$ & $12.5 \pm 0.72$ & $11 \pm 1.44$ \\
\hline SA31 & hosp & MSSA & $6 \pm 0.00$ & $6.5 \pm 0.72$ & $11.5 \pm 0.72$ & $11.5 \pm 0.72$ & $11 \pm 1.44$ \\
\hline SA32 & hosp & MRSA & $6 \pm 0.00$ & $7.5 \pm 0.72$ & $12.5 \pm 0.72$ & $12.5 \pm 0.72$ & $6 \pm 0.00$ \\
\hline SA33 & hosp & MRSA & $6 \pm 0.00$ & $8.5 \pm 0.72$ & $11.5 \pm 0.72$ & $11.5 \pm 0.72$ & $15.5 \pm 0.72$ \\
\hline SA34 & hosp & MRSA & $6 \pm 0.00$ & $8.5 \pm 0.72$ & $10.5 \pm 0.72$ & $11.5 \pm 0.72$ & $13.5 \pm 0.72$ \\
\hline SA35 & hosp & MRSA/MDR & $6 \pm 0.00$ & $8.5 \pm 0.72$ & $12 \pm 1.44$ & $12 \pm 1.4$ & $14.5 \pm 0.72$ \\
\hline SA36 & hosp & MSSA & $6 \pm 0.00$ & $8.5 \pm 0.72$ & $9.5 \pm 0.72$ & $12.5 \pm 0.72$ & $15.5 \pm 0.72$ \\
\hline SA37 & com & MSSA & $6 \pm 0.00$ & $8 \pm 0.00$ & $10.5 \pm 0.72$ & $11.5 \pm 0.72$ & $29.5 \pm 0.72$ \\
\hline SA38 & com & MSSA & $6 \pm 0.00$ & $8.5 \pm 0.72$ & $11 \pm 0.00$ & $13 \pm 0.00$ & $13 \pm 1.44$ \\
\hline SA39 & hosp & MRSA/MDR & $6 \pm 0.00$ & $10.5 \pm 0.72$ & $12 \pm 0.00$ & $13 \pm 0.00$ & $14.5 \pm 0.72$ \\
\hline SA40 & hosp & MRSA/MDR & $6 \pm 0.00$ & $9 \pm 0.00$ & $11 \pm 0.00$ & $11 \pm 0.00$ & $11 \pm 1.44$ \\
\hline S. aureus ATCC 25923 & SR & MSSA & $6 \pm 0.00$ & $8.5 \pm 0.72$ & $10.5 \pm 0.72$ & $12 \pm 1.44$ & $31 \pm 1.44$ \\
\hline
\end{tabular}

Table 6: Diameters of inhibition zone (mm) of Kalanchoe crenata extracts.

Amox: Amoxicillin; com: Community-acquired S. aureus; hosp: hospital-acquired S. aureus.

nigritana (Table 9). The MIC ranged from 0.625 to $2.5 \mathrm{mg} / \mathrm{ml}$ and MBC from 2.5 to $5 \mathrm{mg} / \mathrm{ml}$ for Kalanchoe crenata extracts (Table 8). According to the results, Kalanchoe crenata seems to have bactericidal effects toward all the strains $(1<\mathrm{MBC} / \mathrm{MIC} \leq 4)$. For Mitragyna inermis and Vetiveria nigritana, the intrinsic activity of the extracts was variable. A bacteriostatic effect was recorded with some strains $(8<\mathrm{MBC} / \mathrm{MIC}<16)$ while bactericidal effect was observed with other strains $(1<\mathrm{MBC} / \mathrm{MIC} \leq 4)$.

\section{Discussion}

Antimicrobial susceptibility testing showed that most of the strains $(50 \%)$ of the MRSA were multidrug-resistant. These results confirm the preponderance of antibacterial resistance of Staphylococcus aureus strains. Similar results have been observed by Gupta., et al. [22], Kengne., et al. [23], Garoy., et al. [24], Ibrahim., et al. [25] and Sinda., et al [26]. Nowadays, MRSA has become a

\begin{tabular}{|c|c|c|c|c|c|}
\hline Strains & Strain type & M. inermis extracts & MIC (mg /ml) & $\mathrm{MBC}(\mathrm{mg} / \mathrm{ml})$ & MBC/MIC \\
\hline \multirow[t]{2}{*}{ S. aureus ATCC 25923} & \multirow[t]{2}{*}{ MSSA } & Oven-dried extract & 1.25 & 10 & 8 \\
\hline & & Lyophilized extract & 0.625 & 10 & 16 \\
\hline \multirow[t]{2}{*}{ SA1 } & \multirow[t]{2}{*}{ MRSA/MDR } & Oven-dried extract & 1.25 & 5 & 4 \\
\hline & & Lyophilized extract d & 2.5 & 5 & 2 \\
\hline \multirow[t]{2}{*}{ SA3 } & \multirow[t]{2}{*}{ MRSA/MDR } & Oven-dried extract & 2.5 & 5 & 2 \\
\hline & & Lyophilized extract & 2.5 & 5 & 2 \\
\hline \multirow[t]{2}{*}{ SA35 } & \multirow[t]{2}{*}{ MRSA/MDR } & Oven-dried extract & 1.25 & 5 & 4 \\
\hline & & Lyophilized extract & 1.25 & 5 & 4 \\
\hline \multirow[t]{2}{*}{ SA19 } & \multirow[t]{2}{*}{ MRSA } & Oven-dried extract & 1.25 & 5 & 4 \\
\hline & & Lyophilized extract & 0.625 & 5 & 8 \\
\hline \multirow[t]{2}{*}{ SA24 } & \multirow[t]{2}{*}{ MRSA } & Oven-dried extract & 1.25 & 5 & 4 \\
\hline & & Lyophilized extract & 0.625 & 5 & 8 \\
\hline \multirow[t]{2}{*}{ SA38 } & \multirow[t]{2}{*}{ MSSA } & Oven-dried extract & 1.25 & 5 & 4 \\
\hline & & Lyophilized extract & 1.25 & 5 & 4 \\
\hline
\end{tabular}

Table 7: MIC and MBC obtained with Mitragyna inermis extracts against S. aureus strains. 
In Vitro Antimicrobial Activity of Crude Extracts from Vetiveria nigritana (benth.) Stapf, Mitragyna inermis (Willd.) Kuntze, Kalanchoe crenata (andr.) Haw. against Methicillin-resistant Staphylococcus aureus

\begin{tabular}{|c|c|c|c|c|c|}
\hline Strains & Strain type & K. crenata extracts & MIC & MBC & MBC/MIC \\
\hline \multirow[t]{2}{*}{ S. aureus ATCC 25923} & \multirow[t]{2}{*}{ MSSA } & Oven-dried extract & 1.25 & 2.5 & 2 \\
\hline & & Lyophilized extract & 1.25 & 2.5 & 2 \\
\hline \multirow[t]{2}{*}{ SA1 } & \multirow[t]{2}{*}{ MRSA/MDR } & Oven-dried extract & 2.5 & 5 & 2 \\
\hline & & Lyophilized extract & 1.25 & 5 & 4 \\
\hline \multirow[t]{2}{*}{ SA3 } & \multirow[t]{2}{*}{ MRSA/MDR } & Oven-dried extract & 0.625 & 2.5 & 4 \\
\hline & & Lyophilized extract & 0.625 & 2.5 & 4 \\
\hline \multirow[t]{2}{*}{ SA35 } & \multirow[t]{2}{*}{ MRSA/MDR } & Oven-dried extract & 1.25 & 2.5 & 2 \\
\hline & & Lyophilized extract & 1.25 & 5 & 4 \\
\hline \multirow[t]{2}{*}{ SA19 } & \multirow[t]{2}{*}{ MRSA } & Oven-dried extract & 2.5 & 5 & 2 \\
\hline & & Lyophilized extract & 1.25 & 2.5 & 2 \\
\hline \multirow[t]{2}{*}{ SA24 } & \multirow[t]{2}{*}{ MRSA } & Oven-dried extract & 2.5 & 5 & 2 \\
\hline & & Lyophilized extract & 1.25 & 5 & 4 \\
\hline \multirow[t]{2}{*}{ SA38 } & \multirow[t]{2}{*}{ MSSA } & Oven-dried extract & 1.25 & 5 & 4 \\
\hline & & Lyophilized extract & 1.25 & 5 & 4 \\
\hline
\end{tabular}

Table 8: MIC and MBC obtained with Kalanchoe crenata extracts against S. aureus strains.

\begin{tabular}{|c|c|c|c|c|c|}
\hline Strains & Strain type & V. nigritana extracts & MIC & MBC & MBC/MIC \\
\hline \multirow[t]{2}{*}{ S. aureus ATCC 25923} & \multirow[t]{2}{*}{ MSSA } & Oven-dried extract & 1.25 & 2.5 & 2 \\
\hline & & Lyophilized extract & 1.25 & 2.5 & 2 \\
\hline \multirow[t]{2}{*}{ SA1 } & \multirow[t]{2}{*}{ MRSA/MDR } & Oven-dried extract & 1.25 & 10 & 8 \\
\hline & & Lyophilized extract & 1.25 & 5 & 4 \\
\hline \multirow[t]{2}{*}{ SA3 } & \multirow[t]{2}{*}{ MRSA/MDR } & Oven-dried extract & 2.5 & 10 & 4 \\
\hline & & Lyophilized extract & 1.25 & 5 & 4 \\
\hline \multirow[t]{2}{*}{ SA35 } & \multirow[t]{2}{*}{ MRSA/MDR } & Oven-dried extract & 0.625 & 2.5 & 4 \\
\hline & & Lyophilized extract & 1.25 & 5 & 4 \\
\hline \multirow[t]{2}{*}{ SA19 } & \multirow[t]{2}{*}{ MRSA } & Oven-dried extract & 1.25 & 5 & 4 \\
\hline & & Lyophilized extract & 1.25 & 2.5 & 2 \\
\hline \multirow[t]{2}{*}{ SA24 } & \multirow[t]{2}{*}{ MRSA } & Oven-dried extract & 0.625 & 5 & 8 \\
\hline & & Lyophilized extract & 1.25 & 10 & 8 \\
\hline \multirow[t]{2}{*}{ SA38 } & \multirow[t]{2}{*}{ MSSA } & Oven-dried extract & 1.25 & 10 & 8 \\
\hline & & Lyophilized extract & 1.25 & 5 & 4 \\
\hline
\end{tabular}

Table 9: MIC and MBC obtained with Vetiveria nigritana extracts against S. aureus.

pathogen bacterium with alarming therapeutic problems. This is probably related to its rapid spread and its capacity to acquire resistance to commonly used antibiotics. It would be necessary to search for new active molecules that could limit the diffusion of these MRSAs and ensure the inhibition of their growth without developing resistance to them. Medicinal plants have been widely used to treat a variety of infectious and non-infectious ailments. According to one estimate, $25 \%$ of the commonly used medicines contain compounds isolated from plants [27]. Most of medicinal plants, are well known for their antibacterial, antifungal, antiviral properties [28]. These medicinal plants could be the sources of the treatment of SARM related infections and solve the problem of staphylococcal resistance to current antibacterial agents.

The present study was designed to evaluate the antistaphylococcal properties of extracts from three plants medicinal plants. 
The highest yield of plant extract was obtained from Kalanchoe crenata $(2.72 \%)$ followed by Mitragyna inermis $(2.63 \%)$ while Vetiveria nigritana gave the lowest extract yield. These yields were similar to those obtained by Djoko., et al. [29] with Kalanchoe crenata and those obtained by [29,30] and [31] respectively with Vetiveria nigritana. The differences between yields could be due to the differences of extraction methods, the extraction time, the nature of the extract or the part of the plant used.

In the present study, the antimicrobial activity of extracts of three diverse medicinal plants prepared in different solvents was evaluated against $S$. aureus strains. The extracts showed zones of inhibition which prove the existence of antimicrobial activity against all the MRSA tested. Prior studies have showed the antibacterial activities of Mitragyna inermis, Vetiveria nigritana, Kalanchoe crenata against $S$. aureus strains $[30,32,33]$. The highest antimicrobial activity was observed with ethanol extracts of the three plants. No antimicrobial activity was observed the decoction extracts in this study. These results suggest that antimicrobials activity depend of the solvent used for the extraction. Previous research has shown that ethanol was a better solvent than water for active compounds extraction [34]. In fact, the organic solvents have better results as compared to water $[35,36]$. The overall findings supported the existence of antibacterial metabolites in the crude extract of Mitragyna inermis, Vetiveria nigritana, Kalanchoe crenata against MRSA strains and MRSA/MDR strains.

The range of MIC and MBC of the different extracts observed was 0.625 to $10 \mathrm{mg} / \mathrm{ml}$ for Mitragyna inermis extracts. Similar MIC $(0.625 \mathrm{mg} / \mathrm{ml})$ were obtained with the alkaloids of Mitragyna inermis against Staphylococcus aureus strains [32]. Similar results were recorded with the ethanol extracts of Vetiveria nigritana. However, MIC obtained with essential oils from Vetiveria nigritana $(800 \mathrm{ug} / \mathrm{ml})$ was lower than MIC obtained with solvent extracts [37]. However, the extracts of Mitragyna inermis and Vetiveria nigritana showed bacteriostatic and bactericidal effects against the studied strains. The extracts of Kalanchoe crenata seem to have bactericidal effects on the studied strains. The variation in the antimicrobial activities may be attributed to differences in the time of harvest [38], the developmental stage of plants and the method of extraction [39]. The study have shown that all plants used have overall bactericidal effects on MRSA and that would justify their use in the traditional against infections.

\section{Conclusion}

Results of this study shows in vitro antimicrobial activity of $\mathrm{Mi}$ tragyna inermis, Vetiveria nigritana and Kalanchoe crenata against Staphylococcus aureus isolates including MRSA and MRSA/MDR. Ethanol extracts of these medicinal plants were found to have the strongest and broadest action spectrum. This study brings scientific evidence of the use of these medicinal plants traditionally to combat microbial infections. These plants can be used to formulate a traditional ameliorated herbal medicine against staphylococcal infections and also sources of new actives molecules to fight emerging MRSA.

\section{Acknowledgments}

The authors would like to acknowledge the Biodiversity Information Centre of University Joseph KI-ZERBO for their assistance in plant identification. They sincerely thank the association of traditional healers of Ouagadougou for their collaboration.

\section{Conflict of Interest}

The authors declare that they have no conflicts of interest regarding the data published in this article.

\section{Bibliography}

1. Magill SS., et al. "Multistate point-prevalence survey of health care-associated infections". The New England Journal of Medicine 370.13 (2014): 1198-208.

2. Monegro A.F., et al. "Hospital Acquired Infections". [Updated 2020 Sep 3]. In: StatPearls [Internet]. Treasure Island (FL) StatPearls Publishing (2021)

3. Van Hal SJ., et al. "Vancomycin heteroresistance is associated with reduced mortality in ST239 methicillin-resistant Staphylococcus aureus blood stream infections". PLoS One 6 (2011): e21217.

4. Holland TL., et al. "Vancomycin minimum inhibitory concentration and outcome in patients with Staphylococcus aureus bacteremia: pearl or pellet?". The Journal of Infectious Diseases 204 (2011): 329-331.

5. Tong SYC., et al. "Staphylococcus aureus Infections: Epidemiology, Pathophysiology, Clinical Manifestations, and Management”. Clinical Microbiology Reviews 28.3 (2015): 603-661. 
6. Rhee C., et al. "Incidence and trends of sepsis in US hospitals using clinical vs claims data, 2009-2014". JAMA 318.13 (2017): 1241-1249.

7. Skinner D., et al. "Significance of bacteremia caused by Staphylococcus aureus". Archives of Internal Medicine 68 (1941): 851875 .

8. Lowy FD., et al. "Antimicrobial resistance: the example of Staphylococcus aureus". Journal of Clinical Investigation 111.9 (2003): 1265-1273.

9. Parmar N., et al. "Medicinal plants used as antimicrobial agents : A review". International Research Journal of Pharmacy 3 (2012): 31-40.

10. Kinghorn AD., et al. "The relevance of higher plants in lead compound discovery programs". Journal of Natural Products 74.6 (2011): 1539-1555.

11. Newman DJ., et al. "Natural products as sources of new drugs over the 30 years from 1981 to 2010". Journal of Natural Products 75.3 (2012): 311-335.

12. Atanasov AG., et al. "Discovery and resupply of pharmacologically active plant-derived natural products : A review". Biotechnology Advances 33.8 (2015): 1582-1614.

13. Abreu AC., et al. "Plants as sources of new antimicrobials and resistance-modifying agents". Natural Product Reports 29.9 (2012): 1007-1021.

14. Cheesman MJ., et al. "Developing New Antimicrobial Therapies: Are Synergistic Combinations of Plant Extracts/Compounds with Conventional Antibiotics the Solution?" Pharmacological Reviews 11.22 (2017): 57-72.

15. Gorlenko CL., et al. "Plant Secondary Metabolites in the Battle of Drugs and Drug-Resistant Bacteria: New Heroes or Worse Clones of Antibiotics?" Antibiotics (Basel) 9.4 (2020): 170.

16. Tabish SA. "Complementary and Alternative Healthcare: Is it Evidence-based?". International Journal of Health Science 2.1 (2008): 5-9.

17. WHO. "Who global report on traditional and complementary medicine" (2019)
18. Traore R., et al. "Antimicrobial resistance in Staphylococcus aureus isolates from inpatients and outpatients in Ouagadougou, Burkina Faso". International Journal of Advanced Research 8 (2020): 842-848.

19. CLSI (Clinical and Laboratory Standards Institute). "Performance standards for antimicrobial susceptibility testing; twenty-third informational supplement" (2013).

20. Kitzberger CSG., et al. "Antioxidant and antimicrobial activities of shiitake (Lentinulaedoles) extracts obtained by organic solvents and supercritical fluids". Journal of Natural Products 80 (2007): 631-638.

21. WiegandI., et al. "Agar and broth dilution methods to determine the minimal inhibitory concentration (MIC) of antimicrobial substances". Nature Protocols 3 (2008): 163-175.

22. Gupta BP., et al. "Prevalence of Methicillin Resistant Staphylococcus aureus in Clinical Samples of Teerthankar Mahaveer Medical College Hospital and Research Centre (TMMCH \& RC), Moradabad (UP), India". International Journal of Medical Research and Health Sciences 6.6 (2017): 17-20.

23. Kengne M., et al. "Antibiotic susceptibility patterns of Staphylococcus aureus strains isolated at the Yaounde Central Hospital, Cameroon: a retro prospective study". Pan African Medical Journal 32 (2019): 103.

24. Garoy EY., et al. "Methicillin-resistant Staphylococcus aureus (MRSA): Prevalence and Antimicrobial Sensitivity Pattern among Patients-A Multicenter Study in Asmara, Eritrea". Canadian Journal of Infectious Diseases and Medical Microbiology 6 (2019): 8321834.

25. Ibrahim ES., et al. "Prevalence of multidrug resistance in the Egyptian methicillin-resistant Staphylococcus aureus isolates" African Journal of Biological Sciences 16 (2020): 43-52.

26. Sinda LK., et al. "Prevalence, susceptibility testing and multi drug resistance risk factors to methicillin resistant Staphylococcus aureus in nasal carriage of hospitalized patients and medical staff in selected hospitals in Cameroon". Journal of Microbiology and Antimicrobials 12.2 (2020): 42-51. 
27. Mukhtar M., et al. "Antiviral potentials of medicinal plant". Virus Research - Journal 131.2 (2007): 111-120.

28. Joshi B., et al. "Antibacterial, Antifungal, Antiviral, and Anthelmintic Activities of Medicinal Plants of Nepal Selected Based on Ethnobotanical Evidence". Evidence-Based Complementary and Alternative Medicine 2020 (2020): 1043471.

29. Djoko E., et al. "Formulation d'un médicament traditionnel amélioré à base de Kalanchoe crenata (Andr) haw». Hegel 4.4 (2016): 380-387.

30. Semde Z., et al. "Chemical composition, antioxidant and antimicrobial activities of the essential oil of Vetiverianigritana (Benth.) Stapf roots from Burkina Faso". Journal of Applied Biology and Biotechnology 5 (2017): 29-36.

31. Khalil MAL., et al. "Analysis of the essential oil of Vetiverianigritana (Benth.) Stapf root growing in Sudan". Journal of Medicinal Plant Research 5.32 (2011): 7006-7010.

32. Zongo C., et al. "In vitro Antibacterial Properties of Total Alkaloids Extract from MitragynaInermisc (Willd.) O. Kuntze, a West African Traditional Medicinal Plant". Asian Journal of Plant Sciences 8 (2009): 172-177.

33. Sahin AZ., et al. "Antimicrobial activity of natural compounds from Kalanchoe crenata against pathogenic bacteria". Clinical Microbiology and Infectious Disease 4 (2019): 1-4.

34. Traoré Y., et al. "Recherche des activités antifongique et antibactérienne des feuilles d'Annona senegalensis Pers. (Annonaceae)". Journal of Applied Biosciences 58 (2012): 4234-4242.

35. Rawani A., et al. "Evaluation of antimicrobial properties of four plant extracts against human pathogens". Asian Pacific Journal of Tropical Biomedicine 1.1 (2011): S71-75.

36. Edayadulla N., et al. "Antibacterial activity of various stem extracts of Dalbergia coromandeliana". Asian Pacific Journal of Tropical Biomedicine 2.3 (2012): S1388-1391.

37. Champagnat P., et al. "New compounds from the essential oil of Vetiverianigritana roots from Mali". Flavour and Fragrance Journal 22 (2007): 488-493.
38. Celikel N., et al. "Antimicrobial properties of some essential oils against somepathogenic microorganisms". Czech Journal of Food Sciences 26 (2008): 174-181.

39. Manilal A., et al. "Potential in vitro antimicrobial efficacy of Antibacterial activity of various stem extracts of Dalbergia coromandeliana. (Hook F)". Asian Pacific Journal of Tropical Biomedicine 4 (2014): 25-29.

\section{Assets from publication with us}

- Prompt Acknowledgement after receiving the article

- Thorough Double blinded peer review

- Rapid Publication

- Issue of Publication Certificate

- High visibility of your Published work

Website: https://www.actascientific.com/

Submit Article: https://www.actascientific.com/submission.php Email us: editor@actascientific.com

Contact us: +919182824667 